Abstract 50 Table 2 Troponin status and mortality

\begin{tabular}{lll}
\hline & Alive, $\mathbf{n}$ & Dead, $\mathbf{n}$ \\
\hline Troponin positive & 58 & 66 \\
Troponin negative & 220 & 58 \\
\hline
\end{tabular}

showed that survival of COVID-19 patients was significantly higher in those with a negative troponin $(\mathrm{p}=3.23 \times 10-10)$ compared to those with a positive troponin. A Mann Whitney $\mathrm{U}$ test showed that initial troponin was significantly higher in those who died ( $p=2.24 \times 10-12)$ compared to those who were alive. Mean initial troponin was 89.8 (95\% CI 43.1 136.5). In the multivariate logistical regression, lung disease, age, troponin positivity and CPAP were all significantly associated with death, with an AUC of 0.8872 , sensitivity of 0.9004 and specificity of 0.6292 for the model. Within this model, troponin positivity was independently associated with short term mortality (OR 3.23 , 95\% CI 1.53-7.16, $\mathrm{p}=0.00278)$.

Conclusions We demonstrated an independent association between troponin positivity and increased short-term mortality in COVID-19 in a London district general hospital. The mechanisms implicated in myocardial injury in COVID-19 are not fully understood but are likely multi-factorial.

Conflict of Interest Nil

\section{HIGHLY SENSITIVE TROPONIN ASSAY AS A SINGLE SERUM TEST IN PATIENTS PRESENTING TO A RAPID ACCESS CHEST PAIN CLINIC IN NON-TEACHING HOSPITAL IN THE UK}

Indra Ramasamy. Worcester Royal Hospital, Worcester, UK

10.1136/heartjnl-2021-BCS.51

Background In the UK, rapid access chest pain clinics (RACPC) ensure that patients classified as stable angina in the community can be assessed by a specialist within the hospital system. We report, for the first time, the use of a highly sensitive' troponin assay in the assessment and diagnosis of patients with suspected stable angina, presenting to a RACPC in a non-teaching hospital,

Methods One hundred and seventy two patients admitted to the RACPC were assessed and followed up according to local hospital protocol. Serum cardiac troponin was measured by the 'highly sensitive' cardiac troponin I assay (uscTnI) which had a detection limit of $0.12 \mathrm{ng} / \mathrm{L}$, upper reference limit of $8.15 \mathrm{ng} / \mathrm{L}$ and detected uscTnI in $96.8 \%$ of the population. Clinical assessment of patients was by specialist staff blinded to uscTnI results

Results The mean age of patients was 62.1 years (range 23-88 years) of which $51.1 \%$ were women. The proportion of patients, with recorded cardiovascular risk factors were:hypertension (51\%), tobacco users (44\%), dyslipidemia (48\%), diabetes $(27 \%)$, family history of coronary artery disease (CAD (68\%); increased BMI (29\%). Calculated QRISK3 ranged from $1-88 \%$, mean 16\%. Measured cardiac TnI ranged from $0.71-$ $17 \mathrm{ng} / \mathrm{L}$ (non-cardiac chest pain); 0.97-9.7 ng/L (Exercise tolerance test(ETT) without ischemic changes); 1.9-2.6 ng/L(24 h ECG); 0.46-8.6 ng/L(echocardiogram(echo) without abnormalities); 2.46-17.0 ng/L (echo with mild abnormalities); 1.8-9.2 $\mathrm{ng} / \mathrm{L}$ (diagnosed as angina and treated with medication); 1-70 $\mathrm{ng} / \mathrm{L}$ (further review by cardiologist); 1.5-33.2 ng/L(previous cardiac disease); 0.98-3.9 ng/L (Stress echo (normal); 0.58-9.3 ng/L (computerized tomography coronary angiogram(CCTA), CAD negative); 1.1-8.7 ng/L (CCTA, CAD positive); 1.1-2.2 ng/L (angiogram CAD negative); 0.94-49 ng/L (angiogram CAD positive). Receiver operator characteristic curves(ROC) were used to examine diagnostic threshold at different uscTnI values, measured in patients who underwent functional testing (ETT, Echo), CCTA and coronary angiography. At low values of uscTnI $>0.5 \mathrm{ng} / \mathrm{L}$ sensitivity was $100 \%$, suggesting that uscTnI values $<0.5 \mathrm{ng} / \mathrm{L}$ can be used to exclude CAD. At high values $>11.6 \mathrm{ng} / \mathrm{L}$ specificity was $100 \%$, suggesting patients with values $>11.6 \mathrm{ng} / \mathrm{L}$ may require closer evaluation. In patients assigned to coronary angiogram higher concentrations of uscTnI was associated with more severe CAD. In 3/ 22 patients undergoing CCTA a QRISK3 score $<10 \%$ and uscTnI $<1$ ng/L CCTA was normal, suggesting uscTnI can decrease patient numbers selected for CCTA. There was overlap in uscTnI values between patients with and without CAD in the range $0.5-11.6 \mathrm{ng} / \mathrm{L}$, in this range uscTnI, on its own, may not have the same diagnostic potential.

Conclusions The study suggests that uscTnI is of diagnostic value in patients risk-assessed and allocated to CCTA or coronary angiography. ROC curves suggest that diagnostic cut-off values will depend on patient population and their presenting co-morbidity.

Conflict of Interest none

\section{ROUTINE VERSUS CLINICALLY INDICATED USE OF CHEST X-RAYS IN PATIENTS PRESENTING WITH ST- ELEVATION MYOCARDIAL INFARCTION}

${ }^{1}$ Noor Leelo, ${ }^{2}$ Nicky Mortimer, ${ }^{2} \mathrm{NN}$ Townend, ${ }^{2} \mathrm{SN}$ Doshi, ${ }^{3} \mathrm{M}$ Adnan Nadir. 'University of Birmingham Medical School, Birmingham, UK; ${ }^{2}$ Dept of Cardiology, Queen Elizabeth Hospital, Birmingham, UK; ${ }^{3}$ Queen Elizabeth Hospital Birmingham

\subsection{6/heartjnl-2021-BCS.52}

Background National Institute of Clinical Excellence (NICE) guidelines recommend consideration of Chest X-rays (CXRs) in patients presenting with chest pain to exclude non-cardiac causes of chest pains. However, patients with ST-segment elevation myocardial infarction (STEMI) have a clear diagnosis, rendering the rationale for routine CXRs in this cohort of patients unclear. However, this remains a common practice across the health service. This raises the question of whether CXRs should be conducted routinely or only when clinically indicated.

Methods We performed a retrospective study use of CXRs in consecutive patients admitted with STEMI undergoing primary percutaneous coronary intervention in a single UK tertiary cardiac center. We aimed to investigate if CXRs added clinical and diagnostic value by comparing routine vs. clinically indicated use.

Results A total of 122 patients (Mean Age 63土12, 87\% Male) were admitted with STEMI during the study period and 114/ $122(93.4 \%)$ patients received at least one CXR during their in-patient stay. All but 2/114 were portable thus resourceintensive. Of these, $75 / 114(65.8 \%)$ were routine while 39/ $114(34.2 \%)$ were clinically indicated. Although CXRs were performed in almost all the patients, only 56/114 (49.1\%) of patients had the findings of CXRs documented in the clinical records. The diagnostic efficacy for CXR abnormalities was 
significantly higher in the clinically indicated CXR group (76.9\%) rather than the routine CXR group $(2.7 \%)$ ( $\mathrm{p}=$ $<0.001)$. The therapeutic efficacy was $53.8 \%$ for clinically indicated CXRs, whereas the routine CXRs had a therapeutic efficacy of $1.3 \%$ ( $p=<0.001$ ). There was a significant association between CXR findings and whether the CXR requested was routine or not $(\mathrm{X} 2(1)=70.07, \mathrm{p}<0.001)$ and also management changes (X2 $(1)=45.43, \mathrm{p}<0.001)$.

Conclusion Routine CXR in patient admitted with STEMI are often unnecessary and may add very little clinical value. On the other hand, selective post-procedural CXRs have a significantly higher diagnostic and therapeutic yield. Our study questions the rationale behind routine CXRs in the care of patients with STEMIs.

Conflict of Interest None to declare

\section{THE IMPACT OF FRAILTY ON THE OUTCOMES OF PERCUTANEOUS CORONARY INTERVENTION (PCI) IN THE SETTING OF NON-ST ELEVATION MYOCARDIAL INFARCTION}

'Zhihong Yao, 'Simon Thackray, ${ }^{2}$ Anam Khurram. 'Scunthorpe General Hospital, Scunthorpe, UK; ${ }^{2}$ Hull and York Medical School

\subsection{6/heartjnl-2021-BCS.53}

Introduction With the increase in longevity, older and frailer patients represent a growing cohort for percutaneous coronary intervention (PCI). Frail patients represent a high risk subset and have been shown to predict adverse outcomes more reliably than age. Evidence suggests that PCI may be useful in those who are at high risk for recurrent events. However, frail patients are under-represented in clinical trials with only "fitter" elderly people being included and therefore not being representative of the real-world elderly population. The decision making on intervention regarding elderly or frail patients presenting with non-ST elevation myocardial infarction (NSTEMI) is therefore difficult. The aim of the study is to evaluate the outcomes of NSTEMI PCI with age and frailty independently.

Method Frailty scoring was done retrospectively using the CSHA Frailty Scale for patients presenting to the trust from Jan'16 to Oct'16 \& undergoing PCI for NSTEMI. They were stratified according to age $(<80 \& \geq 80$ yrs) and frailty - not frail (score of 1-3) \& frail (4-6). Outcomes were length of hospital stay, complications and all-cause mortality at 1-year. The association between age and each outcome was examined using Spearman's Rank Correlation; this was also done for frailty.

Result Frailty was assessed in 106 patients with a mean age of $73.5 \pm 12$ years, $65 \%$ were males. 73 (69\%) were not frail and $33(31 \%)$ were vulnerable/frail. The RS between age and frailty was 0.41 indicating moderate correlation between the two variables, $20 / 33(61 \%)$ of the frail group were under 80 . Older and frail group both had higher rates of mortality, contrast nephropathy and longer hospital stay. When the entire cohort was analysed using Spearman's, frailty correlated more strongly with mortality $(\mathrm{r}=0.41 ; \mathrm{p}=0.01)$ and length of stay $(\mathrm{r}=0.3 ; \mathrm{p}=0.04)$ than age however, readmission correlated more strongly with age $(r=0.3 ; p=0.02)$. The association between both age and frailty with nephropathy \& bleeding was not statistically significant nor was the relationship between age and length of stay $(p>0.05)$. Readmission was higher in the frail group but not in the elderly due to noncoronary issues in most.

Conclusion Frail patients, even when younger, have similar outcomes to very old patients, particular with respect to death, kidney damage and subsequent long hospital stay in NSTEMI patients. Additionally, frail score appears to be better predictor for hospital readmission rate compared to age alone. Even in those patient under 80 , frailty score appears to predict these with poorer outcomes, suggesting decision based purely on age are invalid.

Conflict of Interest None

\section{CHANGING INCIDENCE AND PROCEDURE COMPLEXITY OF CORONARY ARTERY PERFORATIONS. - A 10-YEAR DATASET}

${ }^{1}$ Hamza Umar, ${ }^{1}$ Ashwin Roy, Mohammed Oshieba, ${ }^{1}$ Peter Ludman, ${ }^{1} J o n a t h a n$ Townend ${ }^{2}$ M. Adnan Nadir, ${ }^{3}$ SN Doshi, 'S Sudhakar George, ${ }^{1}$ Alex Zaphiriou, ${ }^{2}$ Sohail Q Khan. 'Queen Elizabeth Hospital, University Hospital Birmingham, Birmingham, UK; ${ }^{2}$ Queen Elizabeth Hospital Birmingham; ${ }^{3}$ Dept of Cardiology, Queen Elizabeth Hospital, Birmingham, UK

\subsection{6/heartjnl-2021-BCS.54}

Background Coronary artery perforation (CAP) is a life-threatening complication of percutaneous coronary intervention (PCI). This study aims to identify CAP predictors using contemporary data.

Methods Retrospective cohort study on all PCIs performed at the Queen Elizabeth Hospital, Birmingham between January 2010 and October 2020. Patient demographics, comorbidities, modified Ellis perforation class and perforation treatments were collected. The CAP population was split into two cohorts (1 and 2), representing the first and second 5-year period in the decade.

Results During a 10-year period, 9,504 PCI procedures were performed with CAP occurring in 66 cases. According to the Ellis criteria, $4.5 \%$ of CAPs were type I, $13.6 \%$ type II, $45,5 \%$ type III, and $34.8 \%$ type V. Definitive management included balloon inflation only in $27 \%$, covered stents $20 \%$,

\begin{tabular}{|c|c|c|c|}
\hline & $\begin{array}{l}\text { Cohort 1: }(n= \\
23)\end{array}$ & $\begin{array}{l}\text { Cohort 2: }(n= \\
43)\end{array}$ & $\begin{array}{l}\text { Total PCI }(n= \\
9504)\end{array}$ \\
\hline Mean Age and Standard & $69.3+/-11.3$ & $70.6+/-13.2$ & $64.6+/-12.4$ \\
\hline \multicolumn{4}{|l|}{ Deviation } \\
\hline Male \% & 52.2 & 69.8 & 72.9 \\
\hline Incidence \% & 0.55 & 0.81 & 0.69 \\
\hline Hypertension \% & 69.6 & 65.1 & 59.5 \\
\hline Hypercholesterolaemia \% & 69.6 & 44.2 & 49.7 \\
\hline Chronic Kidney Disease (CKD) \% & 0 & 4.65 & 1.90 \\
\hline Diabetes \% & 13.0 & 28.0 & 26.9 \\
\hline Acute Coronary Syndrome (ACS) & 87.0 & 65.1 & 68.6 \\
\hline \multicolumn{4}{|l|}{$\%$} \\
\hline Сто \% & 8.70 & 25.6 & 6.06 \\
\hline Use of GP IIb/Illa Inhibitors \% & 30.4 & 16.3 & 30.0 \\
\hline Intravascular Ultrasound (IVUS) & 13.0 & 18.6 & 8.86 \\
\hline \multicolumn{4}{|l|}{$\%$} \\
\hline Rotablation \% & 4.35 & 9.30 & 3.23 \\
\hline Tamponade \% & 8.70 & 25.6 & 0.24 \\
\hline Hydrophilic wires \% & 34.8 & 34.9 & 15.0 \\
\hline Mortality \% & 8.70 & 16.3 & 2.58 \\
\hline
\end{tabular}

